\title{
Restorative Treatment Options for Fractured Teeth
}

\author{
Schwedhelm ER* \\ Clinical Associate Professor, Restorative Dentistry, University of Washington, United States
}

${ }^{*}$ Corresponding author: Schwedhelm ER, Clinical Associate Professor, Restorative Dentistry, University of Washington, Box 357456, United States, E-mail: erschwed@uw.edu

Citation: Schwedhelm ER (2016) Restorative Treatment Options for Fractured Teeth. J Oral Health Dent Sci 1(1): 102

Article history: Received: 24 October 2016, Accepted: 09 January 2017, Published: 11 January 2017

\section{Background}

The incidence of fractured teeth seems to be increasing. Fractures normally result from cracks that develop over a period of time until the tooth separates into either buccal or lingual fragments. The diagnosis and early treatment can often prevent needless, extended discomfort and more extensive treatment. Determining the exact nature and extend of the fracture, the restorability of the tooth, and the reversibility of the pulpal response are the dentist's challenge.

The diagnosis and types of treatment modalities will be discussed.

Tooth fractures can occur in many forms, including enamel fractures, enamel-dentin fractures, complicated coronal fractures, crown-root fractures, and root fractures. Of these, coronal tooth fractures are the most common.

\section{Coronal Tooth Fractures}

Coronal tooth fractures represent $26-92 \%$ of all traumatic injuries to the permanent dentition, and can be classified into three groups:

- Craze lines - incomplete fractures of the enamel without loss of tooth substance

- Fractured cusp fractures - fractures not involving the pulp, which can be divided into crown fractures involving enamel only and crown fractures involving dentin

- Split tooth fractures - fractures involving enamel, dentin, and the pulp

- Vertical root fractures - fractures involving the root system

\section{Identification of Coronal Tooth Fractures}

Diagnosis of a cracked tooth can often be difficult since it depends on the affected individual's description of their symptoms. The most common complaint it acute pain when pressure is applied or during mastication. It is important to isolate the pain sensation during mastication to accurately identify the crack lines and severity of the lesion. To assist in the identification, an instrument can be placed over each suspected tooth, while the patient bites on and then releases the biting pressure. Sharp pain on release of pressure will usually identify the offending tooth. This may similarly be accomplished using orangewood sticks, cotton rolls, and instruments such as Fracfinder and Tooth Slooth, which can provide simple and sure diagnosis of the cracked tooth through more accurate delivery of force. In addition, translumination and caries indicator solutions may show loss of continuity in the tooth (Figure 1).

\section{Treatment of Coronal Tooth Fractures}

Craze Lines do not require treatment unless they are severe.

Cusp fractures require treatment in which can follow two possible approaches. The first involves either full crowns or onlays. The second, less invasive treatment involves replacing or placing restorations by bonding the teeth with resin alone or in combination with composite resin, porcelain or bonded amalgam. Another alternative is the placement of a glass ionomer or a hybrid glass ionomer.

Depending on the amount of hard tooth substance loss and soft tissue involvement, different restorative modalities are possible. Priority is always given to the preservation of pulp vitality and sealing the dentin tubules. Treatment may consist of: 
- grinding and/or polishing the fractured enamel for minimal tissue loss

- periodontal crown lengthening

- orthodontic extrusion

- dentin sealing with or without pulp-protection capping

- endodontic treatment

- adhesive fragment reattachment; direct or indirect composite built-up of the missing hard tissues

- extended or prosthetic restoration of the tooth with veneers or crowns

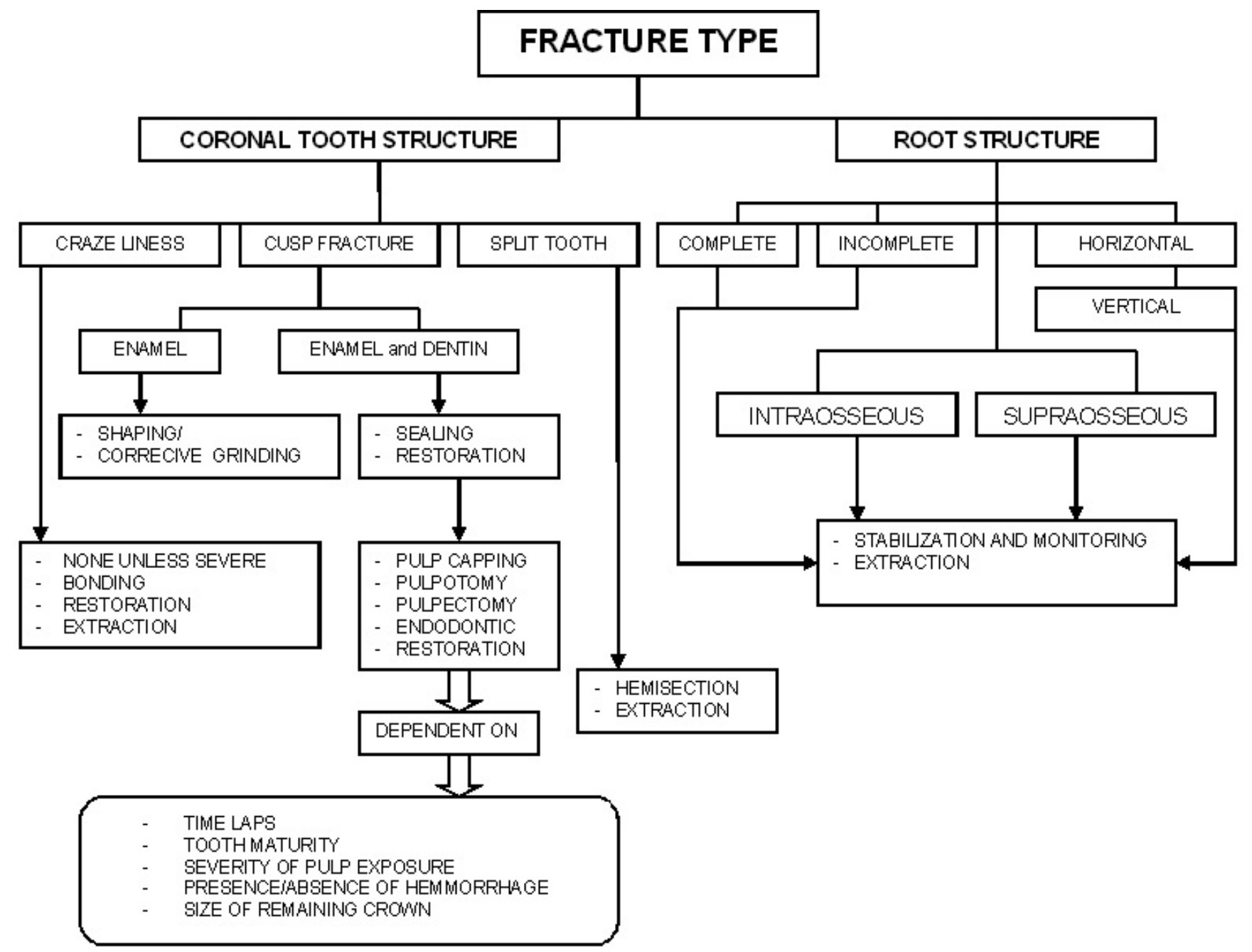

Figure 1: Summary of tooth fractures by type and associated treatment

Treatment of coronal tooth fractures involving only the enamel consists of shaping the sharp edges and/or correctively grinding the tooth. For uncomplicated crown fractures with dentin exposure, measures should be taken to apply a protective material (e.g., calcium hydroxide) over exposed dentin in order to occlude external irritants, such as acid etchant or resin, from causing pulpal inflammation. An acid-etched composite resin is the restoration of choice. Other treatments include immediate crowning of the fractured tooth with a metallic/acrylic crown or an orthodontic band, or permanent treatment with a porcelain jacket crown, porcelain -fused-to-gold crown or cast onlay.

In complicated crown fractures, treatment depends on the maturity of the tooth, the time lapse between the accident and the treatment, the severity of pulp exposure, the presence or absence of hemorrhage, and the size of the remaining crown. Based on these determinants, treatment may involve pulp capping, pulpotomy, pulpectomy (Endo treatment) and/or restoration. When the exposed pulp has been contaminated from extensive crown loss, or treatment time intervals are greater than 24 hours, complete pulpectomy (Endo treatment) may provide the best insurance against residual infection and avoiding residual damage to underlying permanent teeth.

Split tooth fractures normally require the extraction of the tooth.

\section{Root Structure Fractures}

Root fractures are difficult to diagnose because many of the associated signs and symptoms are similar to those for other dental 
problems. Radiographs are often unable to localize the lesion unless fragment separation has already occurred. Diagnostic methods include use of a bite test, dyes to visualize the cracks, and translumination of the teeth. However, when the crack or fracture extends onto the root structure, prognosis is poor and treatment options are often limited to extraction.

Based on fragment separation, two types of root fractures have been described: 1) when total separation is visible or fragments can be moved independently (complete fracture); and 2) when a fracture occurs in the absence of visible separation (incomplete fracture). When a complete fracture occurs, pain is usually persistent and periodontal examination may reveal the presence of deep pocket localized to the affected tooth. Swelling and symptoms of pain localized to the area of the fractured tooth are commonly described.

Root fractures can also be defined relative to the position of the alveolar crest. Intraosseous fractures are those terminating below the level of the alveolar bone and resulting in periodontal problems, whereas supraosseous fractures do not.

Root fractures are also described according to the direction of fracture lines. Horizontal root fractures usually result from direct physical trauma, while vertical root fractures are more common in endodontically treated teeth, although they have also been reported in non-carious and non-endodontically treated teeth. It has been suggested that spontaneous root fracture in nonendodontically treated posterior teeth is related to repetitive excessive occlusive forces.

The early and accurate diagnosis of tooth fracture is important, and will ensure the necessary treatment needed to restore the vitality and function of the dentition. 